order by Copernicus, before Johannes Kepler and the great Isaac Newton could read any kinematical or dynamical laws from it. Since then, the cosmic laboratory works and grows pushing its limits beyond stars, galaxies, and quasars, bringing a flow of new information about expected and unexpected phenomena. At the present time radio-astronomy and space research discover new properties of matter and energy in pulsars, quasars, active nuclei of galaxies, $X$-ray sources, relict radiation, multiatomic molecules in the interstellar medium. Using the most modern techniques of optics, electronics, computing machinery, the modern astronomy stimulates their development by increasing its demands for uttermost precision and perfection and yields to physics and technology-in return-new data on cosmic phenomena.

After a detailed analysis of the progress in all branches of astronomy over the last three years, made in Sydney at the ordinary General Assembly of the International Astronomical Union, within its more than forty commissions, you-astronomers from all the world-came to this country to discuss specifically the present day achievements in these branches of astronomy which took their origin directly from the work of Copernicus: as modern celestial mechanics, exploration of the planetary system, mysteries of gravity in collapsing cosmic masses or expanding envelopes of aging stars, and modern cosmology. These are the topics of the five symposia of this Extraordinary General Assembly. To see the span of progress in astronomy from Copernicus to the present-day astronomy, one more symposium-the sixth-is devoted to the beginnings of this progress, to the background, the birth, and the non-easy reception of the Copernican ideas. This is the plan of our recollection which could be labelled: 'From Copernicus to the present-day astronomy'. Where could we better remember and visualize the Copernican beginnings of our science and its deeply dramatic story, if not visiting these medieval houses, castles, colleges, cathedrals, and towers along the Vistula river, still existing and witnessing the life of the strange man called Nicolaus Copernicus.

There were several reasons why the Polish Academy of Sciences decided to extend their invitation to all astronomers from all countries to come here and to spend a week on fruitful meditations on the topic: 'From Copernicus to Modern Science'

On behalf of the National Committee for Astronomy of the Polish Academy of Sciences I welcome you in this country and wish you both: scientific use and pleasure.'

\title{
ADDRESS BY THE PRESIDENT OF THE IAU, PROFESSOR L. GOLDBERG
}

'The International Astronomical Union is greatly honored to have been invited by the Polish Academy of Sciences to hold an Extraordinary General Assembly as a tribute to Nicolaus Copernicus during the celebration of the quincentenary of his birth. Here in the country where he lived and worked, we have come to pay our respects to the man who initiated research in the physical sciences while hoping at the same time to find new directions for further development of astronomy.

The five symposia to be held in Warsaw, Torun and Cracow during the next ten days are among the most important organized by the IAU in recent years and may well have a decisive influence on the course of cosmological research over the next few years. On behalf of the Executive Committee, I would like to thank the Academy for their invitation, the symposia organizing committees for generating a brilliant scientific program and the local organizing committee for its masterful organization of the very intricate arrangements required to keep 1000 participants supplied with scientific cultural and material nourishments while moving about from one end to the other of this great and beautiful country.

One of the objects of the International Astronomical Union is to develop astronomy through international cooperation. In this we are following the example of Nicolaus Copernicus who, as Prof. Gingerich has pointed out, had no hesitation in acquiring from abroad the knowledge he needed to build his system and in sharing this knowledge with others of different nationality and religious and political beliefs. Although the years he spent in Italy were primarily for the purpose of studying canon law and medicine, he also rounded out his education in mathematics and astronomy and breathed deeply of the atmosphere of the Renaissance. There is considerable evidence that he be- 
came aware of the heliocentric ideas of Aristarchus during this time when the wisdom of the ancient classical period was being re-discovered. The point I wish to make, however, is that Copernicus was a master of foreign languages and that he could therefore initiate his research in astronomy by making a thorough search of the existing literature. Finally, in what was undoubtedly one of the earliest ventures in international scientific publication, the great De revolutionibus was published in Nuremberg through the initiative of the German mathematician Rheticus to whom Copernicus had given his manuscript. So did science progress through international cooperation in the time of Copernicus as it does today.

In assembling in Poland, the IAU also wishes to recognize the important contributions the astronomers of this country have made to the work of the Union. Poland was one of the first countries to satisfy the requirements for admission to the IAU and took part in the first General Assembly in 1922 under the leadership of Tadeusz Banachiewicz. At present the Polish astronomers are taking an active part in the work of the Executive Committee and that of numerous Commissions and are doing important research in a great many different fields, such as spectroscopy, stellar astronomy, radioastronomy, the interstellar medium, stellar evolution, solar physics and solar terrestrial relations. Their activities represent the extension and continuance of a tradition that began even before the birth of Copernicus. In fact, it is not at all surprising that the geocentric universe should have been overturned by a Pole in 16 th Century Europe. Poland in the 16 th century was one of the most advanced countries in Europe and was already famous for its love and learning and for its Jagiellonian University in Cracow which had been founded by Casimir II the Great in 1364. When Copernicus entered this University in 1491, it was already among the oldest and finest in Europe and the only one that could boast not one but two professors of astronomy.

And so, in assernbling here during this jubilee year of 1973, the IAU recognizes its debt, both to Copernicus for setting mankind on the path of cosmic exploration and to our Polish colleagues for preserving and nourishing one of the world's oldest astronomical traditions. I now declare the Extraordinary General Assembly of 1973 officially open.' 